\title{
On the Effects of Spatial Filtering-A Comparative fMRI Study of Episodic Memory Encoding at High and Low Resolution
}

\author{
Peter Fransson, ${ }^{*}, 1$ Klaus-Dietmar Merboldt, $†$ Karl Magnus Petersson, $\ddagger$ Martin Ingvar, $\ddagger$ and J ens Frahm† \\ *MR Research Center and ¥Cognitive Neurophysiology Research Group R2-01, Department of Clinical Neuroscience, \\ Karolinska Institutet/Karolinska Hospital, Stockholm, Sweden; and †Biomedizinische NMR Forschungs GmbH, \\ Max-Planck-Institut für biophysikalische Chemie, 37070 Göttingen, Germany
}

Received J uly 2, 2001

\begin{abstract}
The effects of spatial filtering in functional magnetic resonance imaging were investigated by reevaluating the data of a previous study of episodic memory encoding at $2 \times 2 \times 4-\mathrm{mm}^{3}$ resolution with use of a SPM99 analysis involving a Gaussian kernel of 8-mm full width at half maximum. In addition, a multisubject analysis of activated regions was performed by normalizing the functional images to an approximate Talairach brain atlas. In individual subjects, spatial filtering merged activations in anatomically separated brain regions. Moreover, small foci of activated pixels which originated from veins became blurred and hence indistinguishable from parenchymal responses. The multisubject analysis resulted in activation of the hippocampus proper, a finding which could not be confirmed by the activation maps obtained at high resolution. It is concluded that the validity of multisubject fMRI analyses can be considerably improved by first analyzing individual data sets at optimum resolution to assess the effects of spatial filtering and minimize the risk of signal contamination by macroscopically visible vessels. 2002 Elsevier Science (USA)
\end{abstract}

\section{INTRODUCTION}

Spatial filtering or "smoothing" of positron emission tomography (PET) and functional magnetic resonance imaging (fMRI) data is commonly used in studies of higher cognitive functions of the human brain. In single subjects, the objective for spatial smoothing is to improve sensitivity by low-pass filtering of image data which, for example, suppresses high frequency spatial noise (matched filter theorem, Rosenfeld and Kak, 1982). In order to derive mean results for a group of subjects, spatial filtering becomes mandatory as it accounts for the inherent variability in cerebral anatomy between subjects (e.g., see Petersson et al., 1999). In

\footnotetext{
${ }^{1}$ To whom correspondence and reprint requests should be addressed. Fax: +46-8-5177-3266. E-mail: Peter.Fransson@cns.ki.se.
}

addition, such image data is further smoothed in the spatial normalization procedures (image interpolation) as part of an intersubject statistical analysis which requires all subjects' brain images to be anatomically transformed into a common coordinate system defined by a stereotactic brain atlas.

When studying cognitive processing with blood oxygenation level dependent (BOLD) fMRI in humans, there are at least two aspects of low spatial resolution and filtering which need to be addressed. First, low resolution combined with low-pass spatial filtering may artificially merge areas of activation that belong to neighboring though separate anatomical structures (Worsley et al., 1996; White et al., 2001). And second, the physiologic substrate underlying BOLD fMRI, that is a perfusion-based alteration of the cerebral blood oxygenation, is not uniformly distributed in the brain. BOLD signal changes mainly reflect activation-induced changes of the intravascular concentration of deoxyhemoglobin in venules $(10-30 \mu \mathrm{m})$, pial veins (100-400 $\mu \mathrm{m})$, and even larger veins, which may be distal from the true site of neuronal activation (e.g., see Haacke et al., 1994). Thus, upon spatial smoothing, macrovascular signal contributions which are commonly observed as small clusters of "activated" voxels in the proximity of MRI-resolved veins, will be blurred and given a more "patchy" appearance in neighboring brain tissue simulating "true" parenchymal activations.

The purpose of this paper was to empirically study these effects of spatial filtering on BOLD fMRI activation maps by comparing high-resolution results for visual episodic memory encoding in humans (F ransson et al., 2001) with a newly performed low-resolution analysis. In the original study we investigated hippocampal, parahippocampal, and ventral occipital regions using $\mathrm{fMRI}$ acquisitions at 1-mm section thickness, which were combined to 4-mm sections for functional mapping. Here, in individual subjects, the low-resolution analysis utilized the statistical parametric mapping (SPM 99) software package (F riston et al., 1995) to gen- 


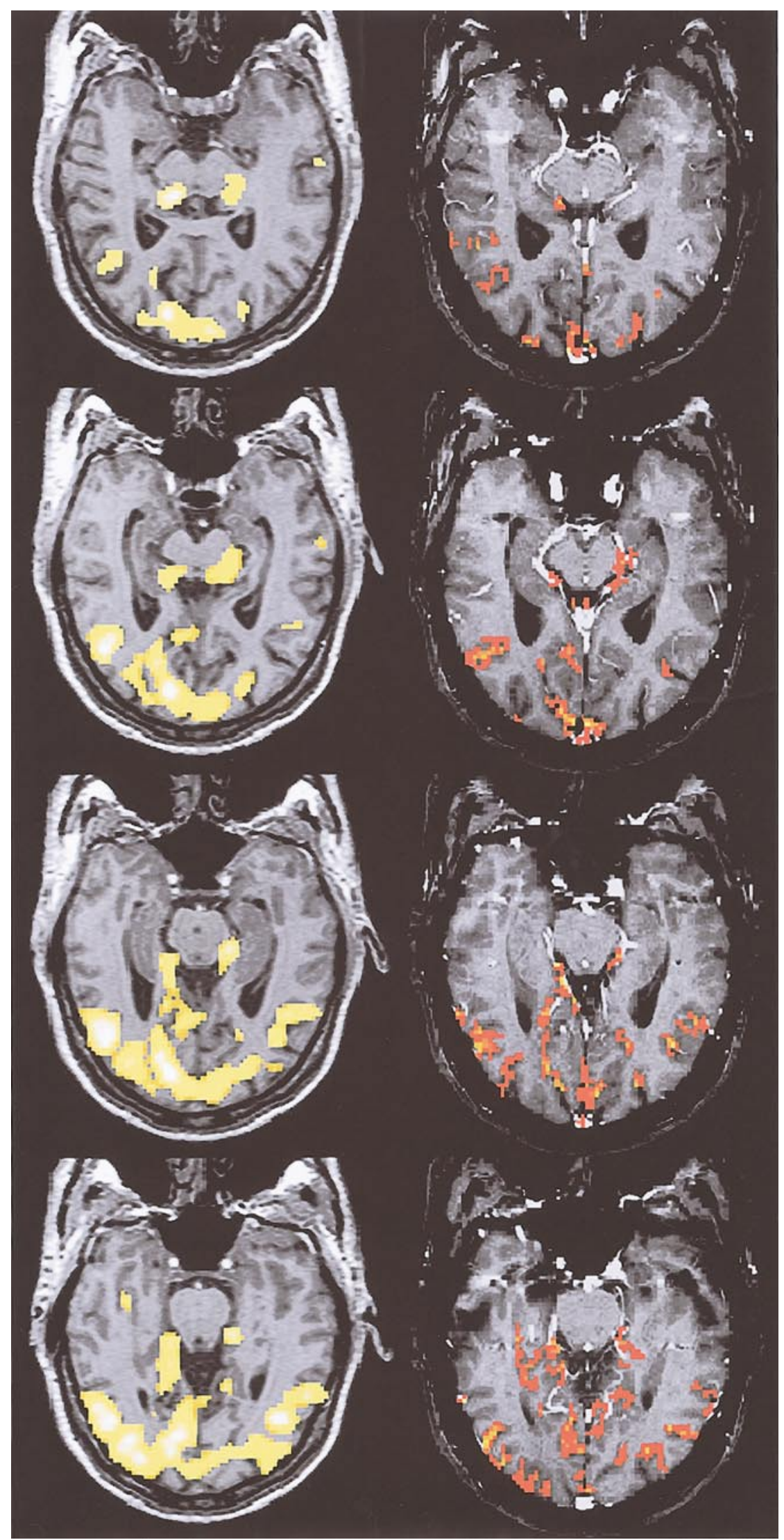

FIG. 1. Activation maps (transverse-to-coronal orientation, single subject, 4 consecutive sections of 4 -mm thickness) obtained for visual episodic memory encoding (left) after temporal and spatial smoothing using an isotropic Gaussian kernel of 8 mm FWHM (SPM analysis) and (right) without temporal or spatial filtering (adapted from Fransson et al., 2001). 
erate activation maps from realigned, coregistered, and spatially smoothed fMRI data. Subsequently, the low-resolution approach was taken one step further by performing a multisubject conjunction or minimum Tfield analysis of all activated brain regions in the medial temporal lobe. This procedure involved a normalization of the individual data sets to the standardized brain template provided by SPM.

\section{MATERIALS AND METHODS}

The comparative analysis included $\mathrm{fMRI}$ acquisitions from eight subjects (25-35 years, mean $29 \pm 3$ years). Written informed consent was obtained from all subjects before the examination. The intentional visual episodic memory encoding task consisted of a presentation of image pairs, which showed a photographed complex visual scene together with the face of the photographer as an insert. A central red cross (fixation point) on a gray screen provided a nonencoding, lowlevel baseline condition. The blocked paradigm employed a 12-s encoding period (three image pairs) alternated with a 18-s control period in a loop of six cycles.

All MRI experiments were carried out at 2.0 T (Siemens Vision, Erlangen, Germany) using the standard head coil. The anatomy of the entire brain was imaged with T1-weighted 3D FLASH (TR/TE = 15/4 ms, flip angle $20^{\circ}$ ), while additional flow-sensitized FLASH images (TR/TE $=70.3 / 6.5 \mathrm{~ms}$, flip angle $60^{\circ}$ ) helped to identify the occurrence of macroscopically visible vessels in the hippocampal region.

BOLD-sensitive T2*-weighted images were acquired using a single-shot, blipped gradient-echo EPI sequence (TR/TE $=2000 / 54 \mathrm{~ms}$ ) with a flip angle of $70^{\circ}$ to minimize T1 saturation and inflow effects. In six subjects, fMRI of the hippocampal region included a 16mm-thick slab covering both left and right hippocampal areas in an oblique transverse orientation parallel to the long axes of the hippocampal bodies. In the remaining two subjects, functional mapping focussed on the right hippocampal region in an oblique sagittal orientation, again along the long axis of the hippocampal body. In either case, high spatial resolution was obtained by acquiring 16 contiguous, interleaved sections with a thickness of $1 \mathrm{~mm}$ and an in-plane resolution of $2 \times 2 \mathrm{~mm}^{2}$ yiel ding a voxel size of $4 \mathrm{~mm}^{3}$. This technique efficiently reduces susceptibility artifacts caused by intravoxel phase dispersion in the inferior part of the medial temporal lobe (Ojemann et al., 1997; Merboldt et al., 2000). Noteworthy, the reduced signalto-noise ratio of a $1 \mathrm{~mm}$ section does not automatically cause a lower functional contrast-to-noise ratio in MRI activation studies. On the contrary, previous work has demonstrated that removal of partial voluming with non-activated brain tissue may significantly enhance the activation-induced BOLD MRI signal change in the
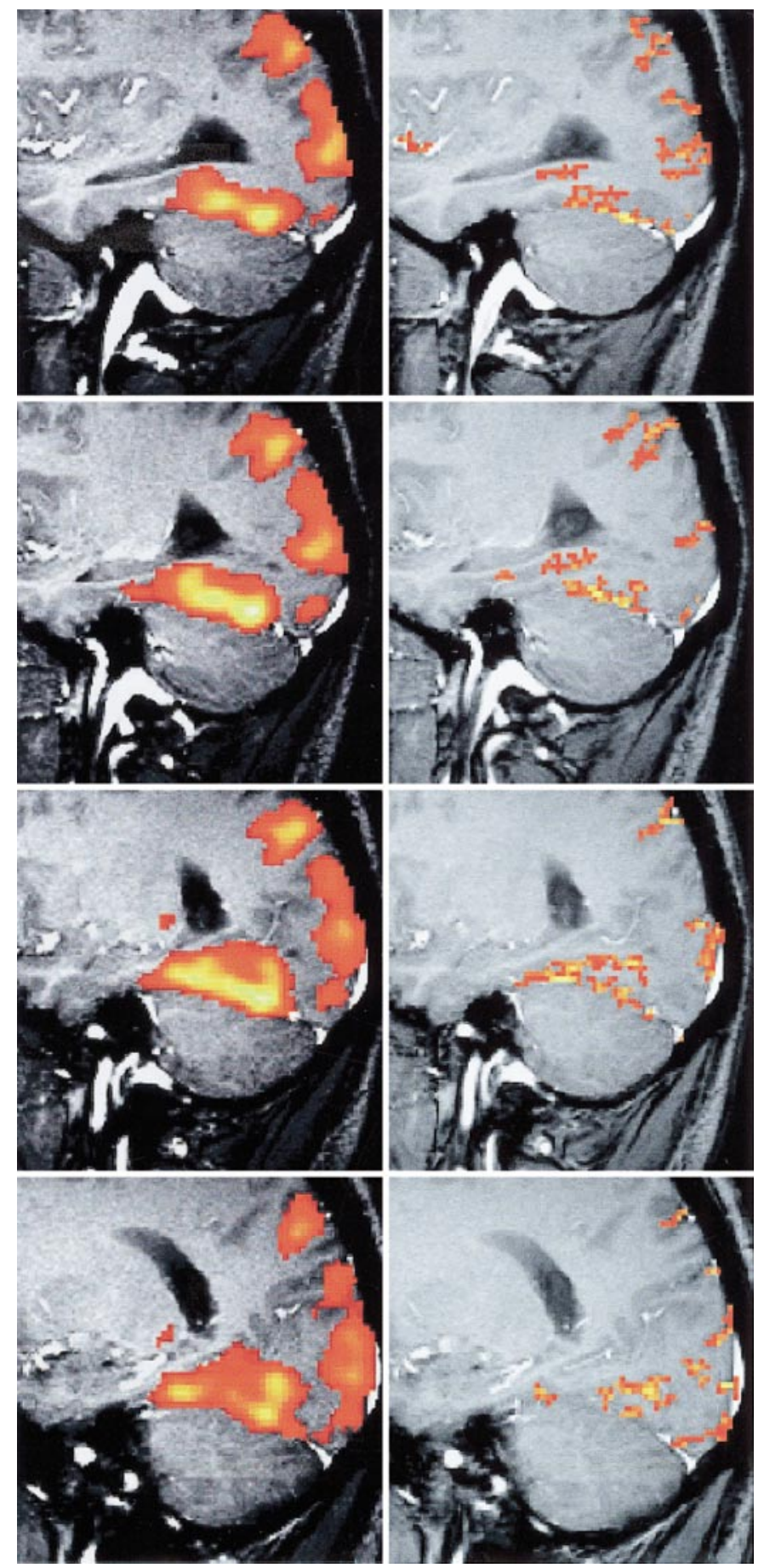

FIG. 2. Activation maps (sagittal-to-coronal orientation, different subject from Fig. 1, 4 consecutive sections of 4-mm thickness) obtained for visual episodic memory encoding (left) after temporal and spatial smoothing using an isotropic Gaussian kernel of 8-mm FWHM (SPM analysis) and (right) without temporal or spatial filtering (adapted from Fransson et al., 2001).

small rim of cortical gray matter (e.g., see Frahm et al., 1993; J esmanowicz et al., 1999). For a full description of the paradigm, the subjects' memory performance, 
and further details of the fMRI acquisition technique see Fransson et al., 2001.

\section{High-Resol ution Analysis}

Apart from grouping the 16 dynamic image sets of 1 $\mathrm{mm}$ section thickness into 4 contiguous sections of 4-mm thickness, the high-resolution approach involved neither temporal nor spatial filtering. BOLD responses related to changes in brain activity were identified by means of a cross-correlation technique which compared the signal intensity time course of each pixel with a box-car reference function shifted by $4 \mathrm{~s}$ with respect to stimulus onset to account for hemodynamic latencies (Bandettini et al., 1993). Maps of correlation coefficients were thresholded in a fully automated and userindependent way by the following procedure. Because task-related effects emerge as positive deviations from an otherwise symmetrical distribution of correlation coefficients, a symmetrized noise distribution is estimated from the histogram of each correlation coefficient map. This allows a rescaling of the correlation coefficients into percentile ranks with respect to the integral of the individual noise distribution. Significant foci of activations were then identified by thresholding the correlation coefficient maps at an error probability of $\mathrm{P} \leq 0.0001$, which corresponds to a percentile rank of $99.99 \%$ of the individual noise distribution. In a second iterative step, the spatial extent of coherently activated pixels was delineated by adding directly neighboring pixels to already accepted foci provided their correlation coefficients exceed a lower threshold chosen to correspond to the $95 \%$ percentile rank of the noise distribution. The procedure follows the ideas outlined in Kleinschmidt et al. (1995).

\section{Low-resolution Analysis}

For the low-resolution approach all processing steps and statistical analyses were carried out using the SPM99 software package (Wellcome Department of Cognitive Neurology, London, UK; www.fil.ion.ud. ac.uk/spm). A correction for subject movements was done for each experimental run by image realignment to the first image vol ume of each session (between-scan movement was less than $1 \mathrm{~mm}$ in all subjects), whereafter a coregistration of the functional images to the anatomical T1-weighted MR brain volume was performed. For single subjects spatial filtering of the raw EPI data sets involved an isotropic Gaussian kernel with a full width at half maximum (FWHM) of $8 \mathrm{~mm}$. In the multi-subject analysis the same filter was applied to co-registered and anatomically normalized images. Temporal smoothing involved low-pass and highpass filtering using cut-off frequencies of $0.25 \mathrm{~Hz}$ and $0.0083 \mathrm{~Hz}$, respectively. The high-pass filter was incorporated as a covariate of no interest (cosine basis functions) in the design matrix. Proportional scaling was applied to account for global confounds. In the multisubject analysis, the fMRI data sets from each subject were anatomically normalized to the standardized SPM brain template using the subjects' coregistered T1-weighted 3-D MRI data. The main effect of interest, that is the encoding versus the control condition, was modelled in SPM by the canonical hemodynamic response function. Voxel-based statistical tests of activated brain regions were performed at $P \leq 0.0001$ (uncorrected) in individual subjects, while activations common to all subjects were identified by means of a conjunction (minimum T-field) analysis at $\mathrm{P} \leq 0.05$ (Friston et al., 1999).

To further substantiate the validity of the low versus high resolution comparison, two complementary SPM analyses were performed (not shown) that either retained the high resolution of the individual data sets or, conversely, applied the low-resolution approach without temporal filtering. The resulting high-resolution SPM maps were in good agreement with those obtained previously by the approach described above. Also the effects of temporal filtering in the low-resolution SPM maps led to only negligible differences. Taken together, these findings suggest that the different approaches yield consistent results in single subjects when using the same degree of image pre-processing.

\section{RESULTS}

Figures 1 and 2 show activation maps for the episodic memory task at low (left columns) and high spatial resolution (right columns) for two different subjects and orientations. Apart from activation in the ventral stream of the occipito-temporal (visual) cortex, the low-resolution maps yield activations in the posterior parahippocampal gyrus and possibly also in the medial-posterior part of the hippocampus proper in transverse sections. Although the corresponding highresolution maps are in qualitative agreement with activations in the ventral occipital cortex and parahippocampal gyrus, a more detailed comparison reveals differences between the two approaches. F or example, the occurrence of a small left temporal activation in the low-resolution maps of Fig. 1 suggests the presence of distributed low levels of activation that escaped significance thresholds in the corresponding high-resolution analysis. More importantly, however, the superposition of the high-resolution maps onto flow-sensitized anatomical images allows a reassignment of apparent activations in the medial-posterior part of the hippocampus proper at low spatial resolution to an area outside the medial border of the hippocampus, in close proximity of the vein of Rosenthal (compare the transverse sections in Fig. 1). In fact, the observation that medial-posterior hippocampus activations on low resolution maps emerged from larger veins outside the 
hippocampus proper was a common finding in all six subjects studied in an oblique transverse orientation.

Apart from the trivial fact that the low-resolution maps display a more blurred "patchy" appearence, a second result for individual subjects is that spatial filtering may cause a merging of activated pixels from neighboring but anatomically (and functionally) separated cortical areas. This is perhaps best demonstrated in the sagittal maps (Fig. 2) which show extended areas of activation in the lower occipital cortex at low resolution, whereas the corresponding high-resolution maps depict distinct activations in the ventral stream of the visual cortex and the more inferiorly located parahippocampal gyrus, respectively.

Taking these findings together we suggest that the activation of the posterior hippocampus proper seen in some low-resolution maps is an effect of spatial filtering during post-acquisition processing which artificially contaminates unaffected tissue with stimuluscorrelated signal changes in larger veins. This is clearly supported by sagittal activation maps at high spatial resolution which unambiguously separate the parahippocampal gyrus from the hippocampus proper. Pertinent maps confine activations in relation to the episodic memory task to the parahippocampal gyrus with no involvement of the hippocampus proper. Nevertheless, strict reasoning can not exclude the possibility of low subthreshold activity in the hippocampus proper that may give rise to BOLD signal changes in larger draining veins.

The results from a multisubject conjunction analysis are summarized in Fig. 3 depicting maximum intensity projections of statistical parametrical maps of visual episodic memory encoding versus rest in an approximate Talairach space. The lower part shows sagittal, coronal, and axial sections of the multisubject activations including the left hippocampus proper $(X=-20$, $\left.Y=-28, Z=-6 ; T=2.41, P=5.1 \times 10^{-8}\right)$. As stated above, this result is not supported by the original individual high-resolution maps.

\section{DISCUSSION}

As far as data for individual subjects are concerned, this comparative empiric study demonstrates two major effects from spatial filtering: (i) insufficient spatial resolution may cause a merging of separate clusters of activated pixels and (ii) small clusters of activated pixels which most likely stem from larger venous vessels become blurred and thus indistinguishable from parenchymal activations. At the multisubject level of analysis, these effects are reinforced by the additional spatial smoothing imposed by multisubject averaging and normalization procedures. As a consequence, the present low-resolution analysis of visual episodic memory encoding leads to a considerable enlargement of activated areas in the medial temporal lobe in compar- ison with the results of a high-resolution analysis. Based on the observed differences in terms of spatial re-assignment and extent of activation, it seems possible that in single subjects and even more likely in a group analysis, the two tested analysis strategies lead to qualitatively different conclusions, that is involvement of the hippocampus proper or not. I n other words, the results of an fMRI study appear to be crucially dependent on the approach chosen for post-acquisition data processing and analysis.

Of course, it may be argued that the effects of spatial filtering may be reduced by selecting a smaller FWHM of the Gaussian kernel in the SPM analysis. This should be possible as the high-resolution images would permit a smaller kernel without violating the requirement that the residual images conform to a lattice approximation of Gaussian random fields as implemented in SPM (see Kiebel et al., 1999). However, because the majority of literature studies employed 8to $15-\mathrm{mm}$ FWHM Gaussian kernels to enhance the sensitivity and reduce the effects of residual anatomical discrepancies as important features in multisubject fMRI studies of higher cognitive functions (see also Hopfinger et al., 2000), the present findings must be considered as representative in terms of the typically achieved spatial resolution.

Notwithstanding the fact that we took precautions to avoid direct inflow effects (Frahm \& al., 1994; Duyn et al., 1994; Glover et al., 1996) by minimizing the degree of T1-weighting, the high-resolution activation maps identify small clusters of activation along the medial borders of the hippocampus proper as BOLD signal contributions from draining veins and not from the capillary bed inside the hippocampus proper (for a detailed description of the vascularization in the hippocampus see Duvernoy, 1998). The actual quantitation of signals from larger vessels versus those from the capillary bed is still an open question in a particular setting (for a further discussion see, e.g., Lai et al., 1999 and Oja et al., 1999). In this respect, a highresolution approach with a voxel size of $4 \mathrm{~mm}^{3}$, which is substantially smaller than typical fMRI acquisitions, turns out to be advantageous, because it reduces partial volume effects with both the macrovasculature and unaffected brain tissue and consequently increases the spatial selectivity and the ability to detect genuine parenchymal activations.

Recently, several schemes have been proposed to ameliorate the poor localization of spatially smoothed activations in multisubject studies. One method uses the concept of scale-space (Worsley et al., 1996) to construct a hierarchical multiscale object-based representation of each individual activation map and thus avoids the limitations of spatial normalization (Coulon et al., 2000). Another method exploits anatomically informed basis functions to incorporate prior information from the individual high-resolution anatomical 

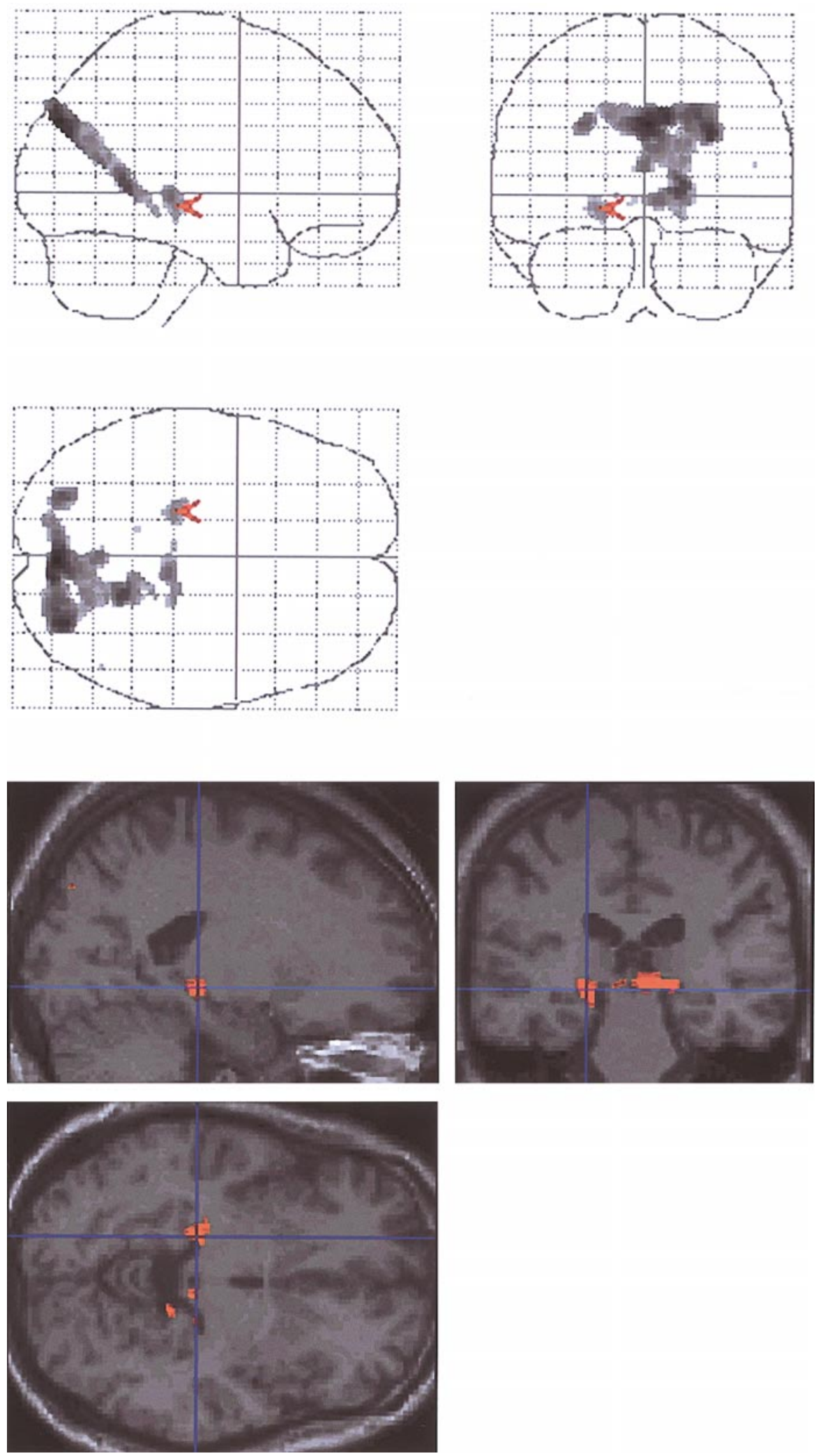

FIG. 3. Multisubject analysis of $\mathrm{fMRI}$ responses to visual episodic memory encoding. (Top) Maximum intensity projections of a statistical parametrical map (SPM $\left[\mathrm{T}_{\min }\right]$, thresholded at $\mathrm{T}=1.26, \mathrm{P} \leq 0.05$, corrected) of activated brain regions in approximate Talairach space, and (bottom) mean activation maps revealing involvement of the left hippocampus proper $\left(X=-20, Y=-28, Z=-6 ; T=2.41, P=5.1 \times 10^{-8}\right)$. 
maps (Kiebel et al., 2000). Similarly, it has been proposed to build upon the equivalence of Gaussian convolution and heat diffusion to achieve an anisotropic smoothing that restricts the spatial extent of activations to the cortical surface (Andrade et al., 2001). F urther promising approaches emerge from the combination of a single-subject analysis with a meta-analysis approach (for a discussion see Petersson et al., 1999). With regard to the present findings, it seems conceivable that problems such as merging of activated regions can be avoided by postprocessing schemes that include prior anatomical information in the spatial smoothing process. On the other hand, the possible inclusion of blurred contributions from larger veins is not fully considered by these methods. We therefore suggest to first assess the physiological origin of the BOLD contrast by analyzing individual fMRI data sets in relation to flow-sensitized structural images or full angiographic maps outlining the respective macrovasculature.

\section{CONCLUSION}

Not unexpectedly, this study confirms for the case of fMRI of episodic memory encoding that spatial smoothing may merge activated areas of anatomically and functionally separated brain structures as well as blur small clusters of activation with a macrovascular origin to such a degree that they appear as parenchymal activations. One possible answer to the question of whether to smooth or not to smooth fMRI data, is to investigate the resulting effects empirically, that is to do both. Hence, a good approach might be to start at high spatial resolution in order to determine potential signal contaminations from venous vessels and to define a suitable smoothing kernel for subsequent processing and statistical inference. In particular, it is recommended to first analyze the fMRI data on an individual basis (note that all subthresholded signals that may contribute to the result of multisubject analyses are missed due to lack of sensitivity at the singlesubject level) before performing a statistical analysis of a group of subjects. The proposed strategy will slightly prolong the MRI scanning session and certainly re quire additional post-acquisition data evaluation. On the other hand, it will markedly reduce the risk of registering activation that results from spatial smoothing rather than from changes in neuronal activity. When following this strategy, it is of course necessary to report inconsistent effects between the two approaches to exclude observer-biased result selection.

\section{ACKNOWLEDGMENT}

P.F. was supported by a grant from the Swedish Brain Foundation.

\section{REFERENCES}

Andrade, A., Kherif, F., Mangin, J . F., Worsley, K. J ., Paradis, A. L., Simon, O., Dehaene, S., LeBihan, D., and Poline, J. B. 2001. Detection of fMRI activation using cortical surface mapping. Hum. Brain Mapp. 12: 79-93.

Bandettini, P. A., J esmanowicz, A., Wong, E. C., and Hyde, J. S. 1993. Processing strategies for time-course data sets in functional MRI of the human brain. Magn. Reson. Med. 30: 161-173.

Coulon, O., Mangin, J . F., Poline, J . B., Zilbovicius, M., Roumenov, D., Samson, Y., Frouin, V., and I. Bloch. 2000. Structural group analysis of functional activation maps. Neurol mage 11: 767782.

Duyn, J . H., Moonen, C. T. W., van Yperen, G. H., de Boer, R. W., and Luyten, P. R. 1994. Inflow versus deoxygenation effects in BOLD functional MRI using gradient echos at 1.5T. NMR Biomed. 7: 83- 88.

Duvernoy, H. M. 1988. The Human Hippocampus. Functional Anatomy, Vascularization and Serial Sections with MRI. Springer Verlag, Berlin.

Fransson, P., Merboldt, K. D., Ingvar, M., Petersson, K. M., and Frahm, J . 2001. Functional MRI with reduced susceptibility artifact: High-resolution mapping of episodic memory encoding. Neuroreport 12: 1415-1420

Frahm, J ., Merboldt, K. D., and Hänicke, W. 1993. Functional MRI of human brain activation at high spatial resolution. Magn. Reson. Med. 29: 139-144.

Frahm, J ., Merboldt, K. D., Hänicke, W., Kleinschmidt, A., and Boecker H. 1994. Brain or vein - Oxygenation or flow? On signal physiology in functional MRI of human brain activation. NMR Biomed. 7: 45-53.

Friston, K. J ., Holmes, A. P., Worsley, K. P., Poline, J . B., Frith, C. D., and Frackowiak, R. S. J . 1995. Statistical parametric maps in functional imaging: A general linear approach. Hum. Brain Mapp. 2: 189-210. [http://www.fil.ion.ucl.ac.uk/spm]

Friston K. J ., Holmes, A. P., Price, C. J ., Büchel, C., and Worsley, K. J . 1999. Multisubject fMRI studies and conjunction analyses. Neuroimage 10: 385-396.

Glover, G. H., Lemieux, S. K., Drangova, M., and Pauly, J . M., 1996. Decomposition of inflow and blood oxygenation level dependent (BOLD) effects with dual-echo spiral gradient-recalled echo (GRE) fMRI. Magn. Reson. Med. 35: 299-308.

Hopfinger, J . B., Büchel, C., Holmes, A. P., and Friston, K. J . 2000. A study of analysis parameters that influences the sensitivity of event-related fMRI analyses. Neurol mage 11: 326-333.

Haacke, E. M., Hopkins, A. L., Lai, S., Buckley, P., Friedman, L., Meltzer, H., Hedera, P., Friedland, R., Klein, S., Thompson, L., Detterman, D., Tkach, J ., and Lewin, J. S. 1994. 2D and 3D High resolution gradient echo functional imaging of the brain: Venous contribution to signal in motor cortex studies. NMR Biomed. 7: 54- 62.

J esmanowicz, A., Biswal, B. B., and Hyde, J. S. 1999. Reduction in GR-EPI intravoxel dephasing using thin slices and short TE. Proc. Intl. Soc. Magn. Reson. Med., 7th Annual Meeting, Philadelphia, p. 1619

Kiebel, S. J., Goebel, R., and Friston, K. J. 2000. Anatomically informed basis functions. Neuroimage 11: 656- 667.

Kiebel, S. J ., Poline, J . B., Friston, K. J ., Holmes, A. P., and Worsley, K.J . 1999. Robust smoothness estimation in statistical parametric maps using standardized residuals from the general linear model. Neurol mage 10: 756-766.

Kleinschmidt, A., Requardt, M., Merboldt, K. D., and Frahm, J . 1995. On the use of temporal correlation coefficients for magnetic resonance mapping of functional brain activation: Individualized 
thresholds and spatial response delineation. Intern. J . Imag. Sys. Technol. 6: 238-244.

Lai, S., Glover, G. H., and Haacke, E. M. 1999. Spatial selectivity of BOLD contrast: Effects in and around veins. In Functional MRI (Moonen, C. T. W., and Bandettini, P. A., Eds.), pp. 221-231. Springer-Verlag, Berlin/Heidelberg/New York.

Merboldt, K. D., Finsterbusch, J ., and Frahm, J. 2000. Reducing inhomogeneity artifacts in functional MRI of human brain activation-Thin sections vs gradient compensation J. Magn. Reson. 145: 184-191.

Oja, J . M. E., Gillen, J ., Kauppinen, R. A., Kraut, M., and van Zijl, P. C. M. 1999. Venous blood effects in spin-echo fMRI of human brain. Magn. Reson. Med. 42: 617-626.

Ojemann, J. G., Akbudak, E., Snyder, A. Z., McKinstry, R. C., Raichle, M. E., and Conturo, T. E. 1997. Anatomical localization and quantiative analysis of gradient refocused echo-planar fMRI susceptibility artifacts. Neurol mage 6: 156-167.

Petersson, K. M., Nichols, T. E., Poline, J . B., and Holmes, A. P. 1999. Statistical limitations in functional neuroimaging II. Signal detection and statistical inference. Phil. Trans. R. Soc. Lond. B 354: 1261-1280.

Rosenfeld, A., and Kak, A. C. 1982. Digital Picture Processing. Academic Press, Orlando, FL.

White, T., O'Leary, D., Magnotta, V., Arndt, S., Flaum, M., and Andreasen, N., 2001. Anatomical and functional variability: The effects of filter size in group fMRI data analysis. Neuroimage 13: 577-588

Worsley, K. J ., Marret, S., Neelin, P., and Evans, A. C. 1996. Searching scale-space for activations in PET images. Hum. Brain Mapp. 4: $74-90$ 